\title{
Extrahepatic Bile Duct Kaposi Sarcoma
}

National Cancer Institute

\section{Source}

National Cancer Institute. Extrahepatic Bile Duct Kaposi Sarcoma. NCI Thesaurus. Code C96951.

A Kaposi sarcoma that arises from the extrahepatic bile ducts. 